\title{
Duty cycle division multiplexing technique for wireless communications
}

\begin{abstract}
A new multiplexing technique which is called duty cycle division multiplexing (DCDM) is presented in this paper. Theoretical and simulation studies have been carried out to evaluate the performance of this technique based on the signal energy and symbol error rate (SER). A wireless channel based on free space propagation model is considered for the simulation study. Two modulation schemes of PSK and QAM are used to evaluate the technique, against the number of users and data rates. Also, the performance of the multiplexing technique is compared with the conventional time division multiplexing (TDM) technique as well as with the multilevel M-ary signaling. The study shows that the energy per bit in the DCDM technique, unlike that of the TDM technique increases with the number of users. The simulation results correspond with the theoretical study in which the DCDM technique has better SER than that of TDM.
\end{abstract}

Keyword: Duty cycle; Multiplexing technique 